\title{
Skill before Knowledge
}

Stanley's Know How defends 'intellectualism': the view that skilled action is guided by propositional knowledge. Abstracting from details, the main argument of the book can be stated like this ${ }^{1}$ :

1 The truth conditions for 'know-how' ascriptions are given by clauses of form

$\ulcorner\mathrm{S}$ knows how to $\Phi\urcorner$ is true in context $\mathrm{c}$ iff $\mathrm{S}$ knows, in c, a proposition of form $<\mathrm{w}$ is a way to $\Phi>$

2 The truth conditions of 'know-how' ascriptions reveal the nature of know-how.

3 An action is skilled iff it manifests the agent's knowledge how to perform it.

4 A skilled $\Phi$-ing manifests the agent's knowledge, with respect to some way $w$, that $w$ is a way to $\Phi$. [From 1, 2, 3]

5 Skilled action is guided by propositional knowledge. [From 4]

I shall consider two objections.

The first concerns the step from $\mathbf{4}$ to $\mathbf{5}$. As far as I can see, Know How contains no argument for this step. Rather, Stanley assumes that with $\mathbf{4}$ granted, $\mathbf{5}$ is inescapable. To overturn this assumption, it suffices to provide an account of how skilled $\Phi$-ing might manifest propositional knowledge without being guided by propositional knowledge. $\S 1$ sketches a route to such an account.

The second objection is directed at 4 . According to this objection, 4 misclassifies something non-conceptual as something conceptual: a skilled $\Phi$-er has a non-conceptual capacity to pick reliable ways to $\Phi ; \mathbf{4}$ misclassifies possession of this non-conceptual capacity. §2 explains this objection and suggests a response that I think advances the debate about singular thought.

\section{Manifestation without guidance}

This section shows how to extend an intuitive, bare-bones account of practical skill into an account of skilled $\Phi$-ing as manifesting, but not guided by, propositional knowledge. I cannot develop the proposal in full detail here. So I shall say just enough to establish two results. Firstly, there is an alternative route to Stanley's 4 - a route independent of Stanley's concern with 'know-how' ascriptions. Secondly, we can agree with Stanley about 4 while rejecting 5. I suggest that each result strengthens the case for 4: the first because it combines with 1-3 to give consistent but disjoint arguments for the same conclusion; the second because $\mathbf{5}$ is objectionable in ways $\mathbf{4}$ alone is not.

\footnotetext{
${ }^{1}$ For a clear statement of 1 see p. 122. For 2 see Ch. $6 \S 4$ esp. pp. 144-145. For 3 see pp. 5, 175. For 4 see For 5 see pp. 14, 23,175
} 
Here is an example to motivate the bare-bones account of practical skill. Suppose Robin Hood and the Sheriff of Nottingham are shooting at a willow wand 250 yards away. Robin is an expert archer, easily capable of this feat in normal conditions. The Sheriff is a poor archer, reliable only given a large, nearby target and no wind. Each intends to hit the target, and takes the most careful aim of which he is capable....

Now consider this question. How should we characterise what Robin (the skilled practitioner) has and the Sheriff (the unskilled participant) lacks? Here is the bare-bones answer that suggests itself. (This is not a full answer. It is what I take to be the uncontroversial fragment every full answer will share.)

The difference in skill is a difference with respect to the relationship between each agent's target-hitting intentions and the behaviours they generate. Robin's actual and likely intentions to shoot are non-lucky selectors of non-lucky means to their fulfilment; the Sheriff's are not. When Robin intends to shoot something, his intention reliably generates a pattern of behaviour which, unless his situation is radically unlucky (a freak gust; a seagull swooping into the arrow's path) will result in success. In contrast, when the Sheriff intends to shoot something, it is very likely that he will fail, and merely lucky if he succeeds.

Generalising and precisifying yield the following:

$\mathrm{S}$ is a skilled $\Phi$-er iff, for appropriate range $\sum$ of situations $\sigma$, in most cases, if $\mathrm{S}$ were to intend to $\Phi$ in $\sigma$, S's intention would select as a means to its fulfilment some $w \in f(\sigma)$, where $f$ is a function taking each $\sigma$ to the set of reliable ways for $\mathrm{S}$ to $\Phi$ in $\sigma$.

For example, $\mathrm{S}$ is a skilled archer iff there is an appropriate range of situations such that, across this range, if $\mathrm{S}$ intends to shoot a target in a situation, $\mathrm{S}$ 's intention will select as a means to its fulfilment some reliable way of hitting the target in the situation.

Now let us move from skilled $\Phi$-ers to skilled $\Phi$-ings. I take it that a 'skilled' action is an action arising in an appropriate way from the skill of a skilled practitioner. Combining this with the suggested account of what it takes to be a skilled practitioner we get

S's $\Phi$-ing in context c is a skilled $\Phi$-ing iff

(i)most of the time, if S intends to $\Phi$ in some $\sigma \in \sum$, S's intention will select some $w \in$ $f(\sigma)$ as a means to its fulfilment (that is, $\mathrm{S}$ is a skilled $\Phi$-er);

(ii) $\mathrm{S}$ intends to $\Phi$ in c;

(iii)the way, $w$, that $\mathrm{S} \Phi$ 's in $\mathrm{c}$ is (appropriately) generated by the selection function mentioned at (i) (that is, S's $\Phi$-ing in c is a $\Phi$-ing-in-way- $w$, and, given S's intention, $\mathrm{S}$ acts in way $w$ because $w \in f(\mathrm{c}))$.

For example, Robin's target-hitting is skilled iff Robin is a skilled archer; he intends to shoot the target; and the behaviour that results in his success is appropriately generated by his intention and his skill - the behaviour is selected by Robin's intention because it will result in reliable target-hitting in the situation.

And now let us focus on (iii) in this formulation - the requirement that S's intention to $\Phi$ generate behaviour $w$ because $w \in f(\mathrm{c})$. (iii) requires that S's acting in 
way $w$ be a response to the fact that $w \in f(\mathrm{c})$. But $\mathrm{S}$ can respond only to facts that $\mathrm{S}$ registers. So (iii) requires that $\mathrm{S}$ register the fact that $w \in f(\mathrm{c})$. But to register the fact that $w \in f(\mathrm{c})$ is to register the fact that $w$ is a reliable way to $\Phi$ in c. And to register this fact is to have a mental state that represents $w$ as a way to $\Phi$ in c.

The claim that $\mathrm{S}$ has a mental state representing $w$ as a way to $\Phi$ in c falls short of the claim that $\mathrm{S}$ grasps the proposition that $w$ is a way to $\Phi$ in $\mathrm{c}$. For not all representation is propositional. But let us grant the step for the moment and see what happens. (Both Stanley and the proponent of the view I am developing here need to defend the claim that the skilled $\Phi$-er's sensitivity to reliable ways of $\Phi$-ing involves grasp of propositions. I shall defend this claim in §2.) of (iii):

The move from 'representation' to 'grasp of a proposition' enables an elaboration

(iii*) S's $\Phi$-ing-in- $w$ in c is a skilled $\Phi$-ing only if a right explanation for the fact that $\mathrm{S}$ $\Phi$ 's-in- $w$ in c appeals to S's grasp of a proposition of form $<w$ is a reliable way to $\Phi$ (in c) $>$.

Given (iii*), we have the claim that a skilled $\Phi$-ing manifests the agent's grasp of a Stanley-esque $<w$ is a reliable way to $\Phi>$ proposition. But is this proposition known by the agent?

Here is a reason to think so.

Note first that the proposition is true. A skilled $\Phi$-ing-in- $w$ in c is a skilled $\Phi$-er's response to the fact that $w \in f(\mathrm{c})$. But $f(\mathrm{c})$ is the set of reliable ways to $\Phi$ in $\mathrm{c}$. So $<w$ is a reliable way to $\Phi$ in $\mathrm{c}>$ is true.

Note also that there are good grounds to regard the agent of a skilled $\Phi$-ing as committed to the truth of the relevant $<w$ is a reliable way to $\Phi>$ proposition. For, on the face of things, the agent's relation to this proposition has the world-to-mind orientation characteristic of 'commitment' propositional attitudes (like knowledge and belief) rather than the mind-to-world orientation characteristic of attitudes like desire and hope. Like belief, and unlike desire, the skilled $\Phi$-er's relation to the $<w$ is a way to $\Phi>$ proposition tracks relevant features of the world (in a context differing with respect to relevant ways to $\Phi$, the skilled $\Phi$-er would not stand in the relation to the same proposition). And like belief, but unlike desire, the agent's relation to the proposition can be undermined by contrary evidence.

Finally, note that it is not just a matter of luck that the agent's commitment is commitment to a true proposition. The agent of a skilled $\Phi$-ing-in- $w$ is committed to the truth of $<w$ is a reliable way to $\Phi>$ because she intends to $\Phi$ and her intention selects $w$ as a reliable means to its fulfilment. Since she is a skilled $\Phi$-er, it is reliably the case that the $w$ selected by her intention to $\Phi$ in fact is a reliable way to $\Phi$. So the story about why a skilled $\Phi$-er is committed to a $<w$ is a reliable way to $\Phi>$ proposition entails that the relevant proposition is very likely to be true.

Putting these observations together, we have the following:

If S's $\Phi$-ing-in- $w$ in c is a skilled $\Phi$-ing, then 
(i) $\mathrm{S}$ is committed to the truth $<w$ is a reliable way to $\Phi$ (in c) $>$;

(ii) This proposition is true;

(iii) $\mathrm{S}$ is not just lucky that the proposition is true.

And now it seems like mere narrow-mindedness to deny S's commitment the status of knowledge. So we have reached our destination: a skilled $\Phi$-ing manifests the agent's knowledge of a $<w$ is a way to $\Phi>$ proposition.

(Note that this path to the claim that skilled $\Phi$-ing manifests knowledge of 'ways' propositions suggests an account of what distinguishes ways from one another: $w=w^{*}$ iff $w$ and $w^{*}$ are reliable across the same range of situations.)

The central contrast between this proposal and Stanley's intellectualism is a contrast in priority of explanation between practical skill and the propositional knowledge manifested by skilled action. Stanwly thinks the possession of practical skill is explained in terms of propositional knowledge:

Intellectualism: knowledge before skill - In a case of skilled $\Phi$-ing, S chooses $w$ as a way to $\Phi$ because $\mathrm{S}$ knows that $w$ is a way to $\Phi ; \mathrm{S}$ is a skilled $\Phi$-er iff $\mathrm{S}$ knows a range of suitable $<w$ is a way to $\Phi>$ propositions.

The view I have sketched reverses Stanley's order of explanation:

Anti-intellectualism: skill before knowledge - S is a skilled $\Phi$-er iff S's intentions to $\Phi$ are non-lucky selectors of non-lucky means to their fulfilment; a skilled $\Phi$-ing manifests propositional knowledge because it is the appropriately generated $\Phi$-ing of a skilled $\Phi$-er.

I suggested earlier that Stanley's 'guidance' claim is objectionable in ways his 'manifestation' claim is not. With the anti-intellectualist view in place, this contention can be substantiated. Some of the most powerful objections to the intellectualist's reading of the 'manifestaion' claim do not arise for the anti-intellectualist's. So an antiintellectualist can agree with Stanley that skilled action manifests propositional knowledge while avoiding many problematic aspects of his proposal. The rest of the section develops a specific instance of this line of thought.

Consider the myriad routes to acquisition of skill. These route include, but are not exhausted by, inborn talent; mindless repetition; unreflective imitation; hypnosis; induction from past attempts; reflection from first principles. The hererogeneity of this list generates an objection to intellectualism. For an account of propositional knowledge needs a justification component. And it is hard to see how the intellectualist can deliver the justification component of the skilled $\Phi$-er's knowledge that $w$ is a way to $\Phi$ while respecting the variety in routes to acquisition of skill.

Here is why. According to intellectualism, the distinctively practical status of the skilled $\Phi$-er's knowledge-that is secureed by how it is manifested: the skilled $\Phi$-er's knowledge-that is essentially manifested in successful $\Phi$-ings. So the status of knowledge-how as a distinctive kind of knowledge-that lies with how the possessor of know-how is disposed to behave. Intellectualism has no resources for a distinctively practical story about how the skilled $\Phi$-er comes by his or her knowledge that $w$ is a way 
to $\Phi$ : the intellectualist explains skill in terms of propositional knowledge, not the other way around. So an intellectualist looking for the justification component of the skilled $\Phi-$ er's propositional knowledge is thrown back on the standard array of accounts of how propositional knowledge is justified.

For example, here is Stanley:

... to know how to catch a fly ball, one must amass enough of the right kind of evidence to know, of a certain way of moving that one thinks of practically, that it is a way that will yield counterfactual success in fly-ball catching. Once this realization has been made, practice leads to ... action without the necessity for reflection. [130]

Stanley stresses that (on his view) a skilled practitioner's knowledge-that is based on a special kind of evidence; that it involves a special ('practical') mode of presentation of ways; and that once knowledge-how is attained, it can guide action unreflectively. But these claims do nothing to secure a distinctive kind of knowledge-how-ish justification: according to Stanley, the justification that secures the skilled practitioner's knowledgethat is just a special case of justification on the basis of evidence.

Now consider again the myriad routes to skill. The intellectualist must either deny that all the routes that seem to belong on the list genuinely are routes to skill, or argue that all of them result in $\langle w$ is a reliable way to $\Phi>$ beliefs for which we can tell something like the justificatory story Stanley tells for the skilled fielder. Though I cannot argue for this claim here, I suggest that neither option promises the intellectualist a happy outcome.

And now consider the anti-intellectualist alternative. On the anti-intellectualist view, the agent of a skilled $\Phi$-ing knows that $w$ is a way to $\Phi$ because (i) s/he is cognitively committed to the proposition $<w$ is a way to $\Phi>$; (ii) this proposition is true; and (iii) given that the agent is a skilled $\Phi$-er, it is not just a matter of luck that s/he is cognitively committed to a true proposition. But the 'luck-eliminating' relation at (iii) is not secured by any evidence the agent might have for the proposition's truth. Rather, it is secured by the agent's skill. On this account, the skilled practitioner's knowledge that $w$ is a way to $\Phi$ is knowledge generated by intention and skill, not knowledge based on evidence.

I suggest that this contrast presents powerful grounds for anti-intellectualism. On an intellectualist view, the justificatory component of the skilled $\Phi$-er's knowledge that $w$ is a way to $\Phi$ must come from the way the skill is acquired. According to antiintellectualism, the justificatory component of the skilled $\Phi$-er's knowledge-that does not come from how the skill is acquired. It comes from the role the skill plays in generating non-lucky fulfilment of intentions to $\Phi$. So, on this view, how you know how to $\Phi$ does not matter from the point of view of whether you do: knowledge-how is an artifact of skill, its possession independent of how the skill is acquired.

\section{Marks of the conceptual}

The second objection I shall consider is directed at Stanley's 4. According to this objection, the skilled $\Phi$-er's sensitivity to which ways of $\Phi$-ing are reliable cannot amount to propositional knowledge, for a mental state can count as propositional 
knowledge iff it is a conceptual mental state, and the skilled $\Phi$-er's sensitivity lacks the characteristic marks of the conceptual.

I shall sketch one reply to this challenge, and close by asking whether this reply is open to Stanley.

First let me make the challenge more precise. One traditional characterisation of conceptual representation of particulars is in terms of grasp of criteria of identity: to think about $o$ you must know what distinguishes $o$ from all $o^{*} \neq o$. Given this traditional view, we can precisify the 'marks of the conceptual' objection as follows.

1 The agent of a skilled $\Phi$-ing-in- $w$ may be unable to articulate or reflect on what distinguishes $w$ from other ways of $\Phi$-ing; recognise $w$ as the same way of $\Phi$-ing employed again; or grasp what it would be for someone else to $\Phi$ in $w$.

2 A subject lacking these capacities does not grasp w's criterion of identity.

\section{So}

3 The agent of a skilled $\Phi$-ing-in- $w$ need not grasp $w$ 's criterion of identity.

\section{Therefore}

4 The agent of a skilled $\Phi$-ing-in- $w$ need not grasp a conceptual representation of $w$.

But

5 In general, grasp of a proposition about a particular involves grasp of a conceptual representation of the particular.

\section{So}

6 The agent of a skilled $\Phi$-ing-in- $w$ need not grasp any $<w$ is a reliable way to $\Phi>$ proposition.

The response to this argument that I want to suggest involves rejection of 5.

To see how this response is avialable, we must reconise an alternative to the framework standardly assumed in the extant philosophical debate about the distinction between conceptual and non-conceptual representation. The extant debate treats this distinction either as holding between either mental states or the contents of mental states. The standard suggestion is then that the propositional attitudes are either conceptual mental states, or mental states with conceptual representational content (depending on choice of 'state' or 'content' account of the conceptual/non-conceptual distinction).

But these standard approaches ignore a third possibility. Rather than, or as well as, contrasting conceptual and non-conceptual mental states and representational contents, we can contrast conceptual and non-conceptual representing relations. For example, suppose we treat the traditional claim that thinking about $o$ involves grasp of 
$o$ 's criterion of identity as a claim about representing relations. Then we can distinguish 'conceptual' from 'non-conceptual' representing relations as follows:

Definition - Relation R between $\mathrm{S}$ and $o$ is a 'conceptual' representing relation iff (a) $\mathrm{S}$ represents $o$ in virtue of standing in R to $o$, and (b) standing in R to $o$ involves grasp of $o$ 's criterion of identity.

Definition - Relation $\mathrm{R}$ between $\mathrm{S}$ and $o$ is a 'non-conceptual' representing relation iff (a) $\mathrm{S}$ represents $o$ in virtue of standing in R to $o$, and (b) standing in R to $o$ involves sensitivity to $o$ rather than any $o^{*} \neq o$, but not grasp of $o$ 's criterion of identity.

Now let us combine this distinction with what I take to be the best contemporary Fregean account of propositions.

Frege thought that propositions are constructed from modes of presentation: the proposition expressed by 'Jack is asleep' is a constructed from a mode of presentation of Jack and a mode of presentation of a function from objects to truth values. This view owes us an explanation of the ontological status of modes of presentation. According to the best contemporary explanation, a mode of presentation is a reification of a representing relation ${ }^{2}$. If you believe that Jack is asleep, you stand in a representing relation to Jack and a representing relation to the function which delivers value TRUE for all and only sleepers as arguments. The Fregean claim that your belief is an attitude to a proposition constructed from modes of presentation is short-hand for the claim that holding the belief involves joint exercise of the representational capacities secured by these representing relations.

This account of propositions combines with the distinction between conceptual and non-conceptual representing relations to deliver a way to resist 5 (the claim that grasp of a proposition about $o$ involves conceptual representation of $o$ ). For if a propositional constituent is a reified representing relation, and there are non-conceptual representing relations, then - absent good reason not to - we should allow that there can be nonconceptual propositional constituents.

I suggest that this line of thought raises the possibility of an improvement on extant accounts of the nature of singular propositions. According to the standard definition, a 'singular' proposition is a proposition containing an object as a constituent. But the central terms in this definition ('contain'; 'constituent') require further explanation. The proposal of this section lets us upgrade the standard definition:

Definition: A 'singular' proposition about $o$ is a proposition grasp of which involves standing in a non-conceptual representing relation to $o$ : a representing relation involving sensitivity to $o$ rather than any $o^{*} \neq o$, but not grasp of $o$ 's criterion of identity.

So here is the response to the 'marks of the conceptual' objection that I suggest. The agent of a skilled $\Phi$-ing-in- $w$ stands in a non-conceptual representing relation to $w$ :

\footnotetext{
${ }^{2}$ Compare Evans The Varieties of Reference ch. $1 \S 4$. Know How ch. $4 \S 1$ contains an illuminating presentation of the same kind of view.
} 
s/he is sensitive to the difference between $w$ and $w^{*}$, but need have no grasp of $w^{\text {'s }}$ criterion of identity.

This proposal raises more questions than I can even state, let alone address, in this venue. But, because I suspect that Stanley will be sympathetic to something like this view, I shall bracket all other issues and close with a question connecting the discussion of this section with the intellectualist/anti-intellectualist contrast from §1: Can Stanley have something like the response to the 'marks of the conceptual' objection that I have proposed?

Here is one reason to think he cannot. We have seen Stanley treating the justificatory story about an instance of know-how as a (special type of) evidence-based story: the skilled $\Phi$-er is justified in taking $w$ to be a way to $\Phi$ because s/he has enough of the right kind of evidence that $w$ is a way to $\Phi$. But it is at least initially plausible that an evidence-based justificatory story can apply only where the proposition supported is fully conceptual. For evidence-based justification requires treating the evidence accrued as relevant to what some particular object is like. And it is hard to see how this will be possible unless the subject grasps the object's criterion of identity.

So there is at least an initial challenge as to whether Stanley can combine an intellectualist view of justification for knowledge-how with a plausible account of how the skilled $\Phi$-er's sensitivity to reliable ways of $\Phi$-ing can count as propositional.

\section{References}

Anscombe, G. E. M. Intention.

Evans, Gareth. The Varieties of Reference.

Stanley, Jason. Know How. New York, Oxford University Press, 2011. 